\title{
Research Article \\ On the Fuzzy Number Space with the Level Convergence Topology
}

\author{
J. J. Font, A. Miralles, and M. Sanchis \\ Institut de Matemàtiques i Aplicacions de Castelló (IMAC), Universitat Jaume I, Campus del Riu Sec. s/n, \\ 12071 Castelló, Spain
}

Correspondence should be addressed to M. Sanchis, sanchis@mat.uji.es

Received 11 October 2012; Accepted 7 November 2012

Academic Editor: Salvador Romaguera

Copyright (C) 2012 J. J. Font et al. This is an open access article distributed under the Creative Commons Attribution License, which permits unrestricted use, distribution, and reproduction in any medium, provided the original work is properly cited.

We characterize compact sets of $\mathbb{E}^{1}$ endowed with the level convergence topology $\tau_{\ell}$. We also describe the completion $\left(\widehat{\mathbb{E}^{1}}, \widehat{\mathfrak{U}}\right)$ of $\mathbb{E}^{1}$ with respect to its natural uniformity, that is, the pointwise uniformity $\boldsymbol{U}$, and show other topological properties of $\widehat{\mathbb{E}^{1}}$, as separability. We apply these results to give an Arzela-Ascoli theorem for the space of $\left(\mathbb{E}^{1}, \tau_{\ell}\right)$-valued continuous functions on a locally compact topological space equipped with the compact-open topology.

\section{Introduction}

Not long after Chang and Zadeh [1] introduced the concept of fuzzy numbers with the consideration of the properties of probability functions, this topic became an active and important area of research because of the development of various theories of fuzzy numbers and their applications in fuzzy optimization, fuzzy decision making, and so forth. In this framework, the study of several types of convergence in fuzzy number spaces plays a central role (see, e.g., $[2,3])$. This paper is a contribution to the study of one of these types of convergence, the so-called level convergence.

Let $F(\mathbb{R})$ denote the family of all fuzzy subsets on the real numbers $\mathbb{R}$, that is, the set of functions $u: \mathbb{R} \rightarrow[0,1]$. For $u \in F(\mathbb{R})$ and $\lambda \in[0,1]$, the $\lambda$-level set of $u$ is defined by

$$
\left.\left.[u]^{\lambda}:=\{x \in \mathbb{R}: u(x) \geq \lambda\}, \quad \lambda \in\right] 0,1\right], \quad[u]^{0}:=\operatorname{cl}_{\mathbb{R}}\{x \in \mathbb{R}: u(x)>0\} .
$$

The fuzzy number space $\mathbb{E}^{1}$ is the set of elements $u$ of $F(\mathbb{R})$ satisfying the following properties:

(1) $u$ is normal, that is, there exists an $x_{0} \in \mathbb{R}$ with $u\left(x_{0}\right)=1$;

(2) $u$ is convex, that is, $u(\lambda x+(1-\lambda) y) \geq \min \{u(x), u(y)\}$ for all $x, y \in \mathbb{R}, \lambda \in[0,1]$; 
(3) $u$ is upper-semicontinuous;

(4) $[u]^{0}$ is a compact set in $\mathbb{R}$.

The $\lambda$-level set $[u]^{\lambda}$ of $u \in \mathbb{E}^{1}$ is a compact interval for each $\lambda \in[0,1]$. We denote $[u]^{\lambda}=$ $\left[u^{-}(\lambda), u^{+}(\lambda)\right]$. Notice that every $r \in \mathbb{R}$ can be considered a fuzzy number since $r$ can be identified with the fuzzy number $\tilde{r}$ defined as

$$
\widetilde{r}(t):= \begin{cases}1 & \text { if } t=r \\ 0 & \text { if } t \neq r\end{cases}
$$

Recall also that Goetschel and Voxman provided the following representation theorem of fuzzy numbers (see [4]).

Theorem 1.1. Let $u \in \mathbb{E}^{1}$ and $[u]^{\lambda}=\left[u^{-}(\lambda), u^{+}(\lambda)\right], \lambda \in[0,1]$. Then the pair of functions $u^{-}(\lambda)$ and $u^{+}(\lambda)$ has the following properties:

(i) $u^{-}(\lambda)$ is a bounded left continuous nondecreasing function on $\left.] 0,1\right]$;

(ii) $u^{+}(\lambda)$ is a bounded left continuous nonincreasing function on $\left.] 0,1\right]$;

(iii) $u^{-}(\lambda)$ and $u^{+}(\lambda)$ are right continuous at $\lambda=0$;

(iv) $u^{-}(1) \leq u^{+}(1)$

Conversely, if a pair of functions $\alpha(\lambda)$ and $\beta(\lambda)$ satisfies the above conditions (i)-(iv), then there exists a unique $u \in \mathbb{E}^{1}$ such that $[u]^{\lambda}=[\alpha(\lambda), \beta(\lambda)]$ for each $\lambda \in[0,1]$.

Actually, the function $u^{-}(\lambda)$ is a nondecreasing function on $[0,1]$, and the function $u^{+}(\lambda)$ is a nonincreasing function on $[0,1]$. This is an easy consequence of condition (iii) of the Goetschel and Voxman representation theorem.

Since $[u]^{\lambda}$ is a compact interval of $\mathbb{R}$ for all $\lambda \in[0,1]$, given two elements $u, v \in \mathbb{E}^{1}$, $[u]^{\lambda}=\left[u^{-}(\lambda), u^{+}(\lambda)\right]$ and $[v]^{\lambda}=\left[v^{-}(\lambda), v^{+}(\lambda)\right]$, we can consider the Hausdorff distance $d$ between $[u]^{\lambda}$ and $[v]^{\lambda}$. It is well known that for compact intervals of the reals, the Hausdorff distance is given by

$$
d\left([u]^{\lambda},[v]^{\lambda}\right)=\max \left\{\left|u^{-}(\lambda)-v^{-}(\lambda)\right|,\left|u^{+}(\lambda)-v^{+}(\lambda)\right|\right\}
$$

The Hausdorff distance allows us to endow $\mathbb{E}^{1}$ with the following metric.

Definition 1.2 (see $[4,5])$. For $u, v \in \mathbb{E}^{1}$,

$$
d_{\infty}(u, v):=\sup _{\lambda \in[0,1]} d\left([u]^{\lambda},[v]^{\lambda}\right)=\sup _{\lambda \in[0,1]} \max \left\{\left|u^{-}(\lambda)-v^{-}(\lambda)\right|,\left|u^{+}(\lambda)-v^{+}(\lambda)\right|\right\},
$$

is a metric on $\mathbb{E}^{1}$. It is called the supremum metric on $\mathbb{E}^{1}$, and $\left(\mathbb{E}^{1}, d_{\infty}\right)$ is a complete metric space.

Definition 1.3. We say that the net $\left\{u_{k}\right\}_{k \in D} \subset \mathbb{E}^{1}$ levelly converges to $u \in \mathbb{E}^{1}$, if $\lim _{k} d\left(\left[u_{k}\right]^{\lambda},[u]^{\lambda}\right)=0$ for any $\lambda \in[0,1]$. 
An easy characterization of level convergence is as follows.

Proposition 1.4. The net $\left\{u_{k}\right\}_{k \in D} \subset \mathbb{E}^{1}$ levelly converges to $u \in \mathbb{E}^{1}$ if and only if $\lim _{k} u_{k}^{+}(\lambda)=u^{+}(\lambda)$ and $\lim _{k} u_{k}^{-}(\lambda)=u^{-}(\lambda)$ for each $\lambda \in[0,1]$.

Notice that a net $\left\{u_{k}\right\}_{k \in D} \subset \mathbb{E}^{1} d_{\infty}$-converges to $u \in \mathbb{E}^{1}$ if and only if $\lim _{k} u_{k}^{+}$converges uniformly to $u^{+}$and $\lim _{k} u_{k}^{-}$converges uniformly to $u^{-}$. Thus, the $d_{\infty}$-convergence implies the $\tau_{\ell}$-convergence. The converse fails to be true (see Example 2.1 in [6]). In [7], Fang and Huang described the topology $\tau_{\ell}$ associated with this level convergence in $\mathbb{E}^{1}$. They showed that $\left(\mathbb{E}^{1}, \tau_{\ell}\right)$ is a Hausdorff, first countable topological space.

By means of the Goetschel-Voxman's representation theorem, we can regard $\left(\mathbb{E}^{1}, \tau_{\ell}\right)$ as a subspace of the product space $\mathbb{R}^{[0,1]} \times \mathbb{R}^{[0,1]}$ (which can be identified with $(\mathbb{R} \times \mathbb{R})^{[0,1]}$ in a canonical way). Indeed, the correspondence $u \in \mathbb{E}^{1} \stackrel{i}{\hookrightarrow}\left(u^{-}, u^{+}\right)$defines a homeomorphism from $\left(\mathbb{E}^{1}, \tau_{\ell}\right)$ into $\left(\mathbb{R}^{[0,1]} \times \mathbb{R}^{[0,1]}, \tau_{p}\right)$, where $\tau_{p}$ denotes the pointwise topology. Thus, basic open sets in $\tau_{\ell}$ are given by

$$
U\left(u,\left\{\lambda_{1}, \ldots, \lambda_{n}\right\}, \epsilon\right):=\left\{v \in \mathbb{E}^{1}: \max _{1 \leq i \leq n}\left\{\left|v^{+}\left(\lambda_{i}\right)-u^{+}\left(\lambda_{i}\right)\right|,\left|v^{-}\left(\lambda_{i}\right)-u^{-}\left(\lambda_{i}\right)\right|\right\}<\epsilon\right\},
$$

for $\left\{\lambda_{1}, \ldots, \lambda_{n}\right\} \subset[0,1], u \in \mathbb{E}^{1}$, and $\epsilon>0$.

In Section 2, we will first prove that the subset of all elements $u$ of $\mathbb{E}^{1}$ such that $u^{-}$and $u^{+}$are continuous is dense in $\mathbb{E}^{1}$, and we will also study compact sets of $\left(\mathbb{E}^{1}, \tau_{\ell}\right)$, giving a characterization to be compared with the one provided in [7]. In Section 3, we will provide a description of the completion $\left(\widehat{\mathbb{E}^{1}}, \widehat{\mathcal{U}}\right)$ of $\mathbb{E}^{1}$ with the pointwise uniformity $\boldsymbol{U}$, and we will show that its underlying topological space is separable, Lindelöf (thus, strongly paracompact) and a Baire space. In the last section, an Arzela-Ascoli theorem for the space of $\left(\mathbb{E}^{1}, \tau_{\ell}\right)$-valued continuous functions will be stated.

\section{Two Useful Results}

Our first result provides a dense subset of $\left(\mathbb{E}^{1}, \tau_{\ell}\right)$. We will consider the subset $\mathbb{C}^{1}$ of $\mathbb{E}^{1}$ given by

$$
\mathbb{C}^{1}:=\left\{u \in \mathbb{E}^{1} \mid u^{-} \text {and } u^{+} \text {are continuous functions }\right\}
$$

Theorem 2.1. $\mathbb{C}^{1}$ is dense in $\left(\mathbb{E}^{1}, \tau_{\ell}\right)$.

Proof. Given a basic open set $U\left(u,\left\{\lambda_{1}, \ldots, \lambda_{n}\right\}, \epsilon\right)$ in $\left(\mathbb{E}^{1}, \tau_{\ell}\right)$ with $\lambda_{i}<\lambda_{i+1}$ for $i=1,2, \ldots, n-1$, consider $\left(u_{1}, u_{2}\right) \in \mathbb{C}^{1}$ where $u_{1}$ is defined as

$$
u_{1}(\lambda):= \begin{cases}u^{-}(0) & \text { if } \lambda=0 \\ u^{-}\left(\lambda_{i}\right) & \text { if } \lambda=\lambda_{i}, i=1,2, \ldots, n \\ u^{-}(1) & \text { if } \lambda=1\end{cases}
$$


and linearly at intermediate values, and $u_{2}$ is defined as

$$
u_{2}(\lambda):= \begin{cases}u^{+}(0) & \text { if } \lambda=0 \\ u^{+}\left(\lambda_{i}\right) & \text { if } \lambda=\lambda_{i}, i=1,2, \ldots, n \\ u^{+}(1) & \text { if } \lambda=1\end{cases}
$$

and linearly at intermediate values. It is an easy matter to check that $\left(u_{1}, u_{2}\right) \in \mathbb{C}^{1}$ and that $\left(u_{1}, u_{2}\right) \in U\left(u,\left\{\lambda_{1}, \ldots, \lambda_{n}\right\}, \epsilon\right)$. Thus, $\mathbb{C}^{1}$ is dense in $\left(\mathbb{E}^{1}, \tau_{\ell}\right)$.

By [8, Theorem 6.1], we know that the topology of pointwise convergence and the topology of uniform convergence coincide in the set of all monotone continuous functions defined on the unit interval. Taking into account these facts and properties (i) and (ii) of Theorem 1.1, it is clear that $\left.\tau_{d_{\infty}}\right|_{\mathbb{C}^{1}}=\left.\tau_{\ell}\right|_{\mathbb{C}^{1}}$ where, as usual, $\tau_{d_{\infty}}$ stands for the topology induced by the metric $d_{\infty}$.

There are several characterizations of compact subsets of spaces of functions $₹$ depending on the topology we endow the space with. For instance, if we deal with the topology of uniform convergence $\tau_{u}$ on some spaces of continuous functions, the AscoliArzela's Theorem asserts that $K \subset\left(\mathcal{F}, \tau_{u}\right)$ is compact if and only if $K$ is pointwise bounded, closed and equicontinuous. Dealing with the pointwise convergence topology $\tau_{p}$ in more general spaces of functions $\mathcal{F}$, it is well known that $K \subset\left(\mathcal{F}, \tau_{p}\right)$ is compact if and only if $K$ is closed and pointwise bounded. Hence, it is clear that closed and pointwise bounded subsets $A \subset \mathcal{F}$ satisfying $\tau_{p}=\tau_{u}$ are also equicontinuous.

Definition 2.2 (see [5]). It is said that $A \subset \mathbb{E}^{1}$ is uniformly support bounded, if there is a constant $C>0$ such that $\max \left\{\left|u^{-}(0)\right|,\left|u^{+}(0)\right|\right\} \leq C$ for all $u \in A$.

As it is customary, we say that a subset $A$ of $\mathbb{E}^{1}$ is pointwise closed, if $A$ is a closed subset of the product space $\mathbb{R}^{[0,1]} \times \mathbb{R}^{[0,1]}$ (or equivalently, of the product space $(\mathbb{R} \times \mathbb{R})^{[0,1]}$.

Theorem 2.3 (compare [7]). A subset $K$ of $\left(\mathbb{E}^{1}, \tau_{\ell}\right)$ is compact if and only if $K$ is pointwise closed and uniformly support bounded.

Proof. Suppose that $K$ is a compact subset of $\left(\mathbb{E}^{1}, \tau_{\ell}\right)$. Then $K$ is compact in $\mathbb{R}^{[0,1]} \times \mathbb{R}^{[0,1]}$, that is, $K$ is pointwise closed and pointwise bounded which implies that $K$ is pointwise closed and uniformly support bounded. Conversely, if $K \subset \mathbb{E}^{1}$ is uniformly support bounded, $K$ is pointwise bounded. Thus, if $K$ is also pointwise closed, $K$ is a compact subset of $\mathbb{R}^{[0,1]} \times \mathbb{R}^{[0,1]}$. Since compactness is an absolute topological property, $K$ is a compact subset of $\left(\mathbb{E}^{1}, \tau_{\ell}\right)$.

Example 2.4. There are examples of $\tau_{\ell}$-closed and uniformly support bounded subsets of $\mathbb{E}^{1}$ which are not $\tau_{\ell}$-compact. Consider a uniformly support bounded Cauchy sequence $\left\{u_{n}\right\}_{n=1}^{\infty}$ in $\left(\mathbb{E}^{1}, \tau_{\ell}\right)$ that does not converge (see, e.g., [9]). Since $\left(\mathbb{E}^{1}, \tau_{\ell}\right)$ is first countable, the set $K=$ $\left\{u_{n}\right\}_{n=1}^{\infty}$ is uniformly support bounded and $\tau_{\ell}$-closed in $\left(\mathbb{E}^{1}, \tau_{\ell}\right)$ but it is not compact.

We also notice that there exist compact metric subsets $K$ of $\left(\mathbb{E}^{1}, \tau_{\ell}\right)$ such that $\left.\tau_{d_{\infty}}\right|_{K} \neq\left.\tau_{\ell}\right|_{K}$. 
Example 2.5. Let $\left\{\left(u_{n}^{-}, v_{n}^{+}\right)\right\}_{n \geq 1}$ be the sequence in $\mathbb{E}^{1}$ defined as

$$
u_{n}^{-}(\lambda)= \begin{cases}1 & \text { for each } \lambda \leq \frac{1}{2} \\ \frac{1}{2} & \text { for each } \lambda>\frac{1}{2}+\frac{1}{n}\end{cases}
$$

extended linearly at intermediate values, and $v_{n}^{+}=0$ for all $n \geq 1$. It is clear that the sequence $\left\{\left(u_{n}^{-}, v_{n}^{+}\right)\right\}_{n \geq 1}$ converges to $\left(u^{-}, 0\right)$ with $u^{-}(\lambda)=1$ if $\lambda \leq 1 / 2$ and $u^{-}(\lambda)=1 / 2$ if $\lambda>1 / 2$. Then $K=\left\{\left(u_{n}^{-}, v_{n}^{+}\right)\right\}_{n \geq 1} \cup\left\{\left(u^{-}, 0\right)\right\}$ is a compact subset of $\left(\mathbb{E}^{1}, \tau_{\ell}\right)$. Since the function $u^{-}$is not continuous, $K$ is not $d_{\infty}$-compact.

\section{Topological and Uniform Properties of $\mathbb{E}^{1}$}

First we prove the following.

Theorem 3.1. $\left(\mathbb{E}^{1}, \tau_{\ell}\right)$ is separable.

Proof. We will denote by CMI (resp., CMD,) the set of all nondecreasing monotone continuous functions (resp., the set of all nonincreasing monotone continuous functions) on $[0,1]$. It is well known that the space $C([0,1])$ of all continuous real-valued functions on $[0,1]$ endowed with the topology of the uniform convergence is separable by the classical StoneWeierstrass theorem. Separability is a hereditary property in the realm of metric spaces, so that the spaces CMI and CMD equipped with the $\tau_{u}$-topology are separable spaces. Since the topologies of uniform convergence and pointwise convergence coincide on $\mathrm{CMI} \times \mathrm{CMD}$, the product space $\left(\mathrm{CMI} \times \mathrm{CMD}, \tau_{p}\right)$ is a separable metric space and, consequently, the space $\mathbb{C}^{1}$ is separable. The result now follows from Theorem 2.1.

The correspondence $u \in \mathbb{E}^{1} \stackrel{i}{\hookrightarrow}\left(u^{-}, u^{+}\right)$is a uniform isomorphism when we consider the natural admissible structure $U$ on $\left(\mathbb{E}^{1}, \tau_{\ell}\right)$ and the pointwise uniform structure (or pointwise uniformity) uniform structure $\mho$ on $\left(\mathbb{R}^{[0,1]} \times \mathbb{R}^{[0,1]}, \tau_{p}\right)$ which has as a base the sets $U\left(\lambda_{1}, \lambda_{2}, \ldots, \lambda_{n}, \varepsilon\right)(n \geq 1, \varepsilon>0)$ of the following form:

$$
\begin{aligned}
& \left\{((f, g),(h, t)) \in\left(\mathbb{R}^{[0,1]} \times \mathbb{R}^{[0,1]}\right) \times\left(\mathbb{R}^{[0,1]} \times \mathbb{R}^{[0,1]}\right):\left|f\left(\lambda_{i}\right)-h\left(\lambda_{i}\right)\right|<\varepsilon,\right. \\
& \left.\quad\left|g\left(\lambda_{i}\right)-t\left(\lambda_{i}\right)\right|<\varepsilon, i=1,2, \ldots, n\right\},
\end{aligned}
$$

where, for all $n \geq 1,\left(\lambda_{1}, \lambda_{2}, \ldots, \lambda_{n}\right)$ runs over $[0,1]^{n}$. Throughout, we shall freely identify, without explicit mention, the uniformity $\mathcal{U}$ with the restriction of the uniformity $\mho$ to $i\left(\mathbb{E}^{1}\right)$. It is a well-known fact that the uniform space $\left(\mathbb{E}^{1}, \mathfrak{U}\right)$ is not complete ([9]). Hence, our next goal is to describe its completion $\left(\widehat{\mathbb{E}^{1}}, \widehat{\mathfrak{U}}\right)$. Remark that a consequence of the previous theorem is the following.

Corollary 3.2. The underlying topological space of $\left(\widehat{\mathbb{E}^{1}}, \widehat{u}\right)$ is separable. 
Theorem 3.3. The completion $\left(\widehat{\mathbb{E}^{1}}, \widehat{\mathcal{U}}\right)$ of the uniform space $\left(\mathbb{E}^{1}, \mathfrak{U}\right)$ is the subspace of $\left(\mathbb{R}^{[0,1]} \times\right.$ $\left.\mathbb{R}^{[0,1]}, \mho\right)$ whose elements $(u, v)$ verify the following properties:

(i) $u$ is a nonincreasing function on $[0,1]$;

(ii) $v$ is a nondecreasing function on $[0,1]$;

(iii) $u(1) \leq v(1)$.

Proof. The uniform space $\left(\mathbb{R}^{[0,1]} \times \mathbb{R}^{[0,1]}, \circlearrowright\right)$ is a product of complete uniform spaces, so that it is complete. Hence $\mathrm{cl}_{\mathbb{R}^{[0,1]} \times \mathbb{R}^{[0,1]}} \mathbb{E}^{1}$ is a complete uniform space containing $\mathbb{E}^{1}$ as dense subset. By [10, Theorem 8.3.12], $\mathrm{cl}_{\mathbb{R}^{[0,1]} \times \mathbb{R}^{[0,1]}} \mathbb{E}^{1}$ can be identified with the completion of $\left(\mathbb{E}^{1}, \mathfrak{U}\right)$.

We move on to the description of $\mathrm{cl}_{\mathbb{R}^{[0,1]} \times \mathbb{R}^{[0,1]}} \mathbb{E}^{1}$. First notice that if $(u, v) \in \mathrm{cl}_{\mathbb{R}^{[0,1]} \times \mathbb{R}^{[0,1]}} \mathbb{E}^{1}$, then $u$ is nonincreasing and $v$ is nondecreasing since a pointwise limit of a net of nonincreasing (resp., nondecreasing, functions is a nonincreasing (resp., a nondecreasing, function. Moreover, since every $\left(u^{-}, u^{+}\right) \in \mathbb{E}^{1}$ satisfies $u^{-}(1) \leq u^{+}(1)$, condition (iii) is an

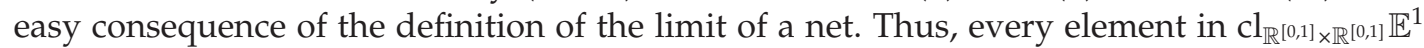
verifies conditions (i)-(iii). The converse follows from an argument similar to the one used in Theorem 2.1.

Corollary 3.4. The uniformity induced by the metric $d_{\infty}$ and the uniformity $u$ induce the same topology on the set $\mathbb{C}^{1}$, but the first is finer than the second.

As we have seen above, the topology $\tau_{d_{\infty}}$ induced by the metric $d_{\infty}$ and the level topology $\tau_{\ell}$ coincide in $\mathbb{C}^{1}$. This question dealing with $\mathbb{E}^{1}$ seems to have received almost no attention in the literature. We borrow from [11] the necessary techniques in order to obtain a characterization of subsets $A \subset \mathbb{E}^{1}$ satisfying that $\left.\tau_{d_{\infty}}\right|_{A}=\left.\tau_{p}\right|_{A}$. Recall that a function $f$ between two first countable spaces $X$ and $Y$ is continuous if and only if $f\left(\lim _{n} x_{n}\right)=\lim _{n} f\left(x_{n}\right)$ for every sequence $\left\{x_{n}\right\}$ in the space $X$. For every $u \in \mathbb{E}^{1}$ and $\lambda \in[0,1[$, we will denote by $u\left(\lambda^{+}\right)$the limit of $u(t)$ when $t \rightarrow \lambda^{+}$. First we need the following helpful result.

Lemma 3.5. If $\left\{u_{n}\right\}_{n=1}^{\infty}$ is a $\tau_{\ell}$-convergent sequence, then for all $\varepsilon>0$ and $\left.\left.\lambda_{0} \in\right] 0,1\right]$, there exists $\delta>0$ such that $u_{n}^{-}\left(\lambda_{0}\right)-u_{n}^{-}(\lambda)<\varepsilon$ and $u_{n}^{+}(\lambda)-u_{n}^{+}\left(\lambda_{0}\right)<\varepsilon$ for all $n \in \mathbb{N}$ whenever $\lambda \in\left[\lambda_{0}-\delta, \lambda_{0}\right]$.

Proof. The proof for $\left\{u^{+}\right\}_{n=1}^{\infty}$ proceeds along the same lines as the proof for $\left\{u^{-}\right\}_{n=1}^{\infty}$ so that we only show our lemma for the sequence $\left\{u^{-}\right\}_{n=1}^{\infty}$. Let $u=\left(u^{-}, u^{+}\right)$be the limit point of $\left\{u_{n}\right\}_{n=1}^{\infty}$. Since $u^{-}$is left-continuous on $(0,1]$ and bearing in mind that $u^{-}$is nondecreasing, there exists $\delta>0$ such that $0 \leq u^{-}(\lambda)-u^{-}\left(\lambda_{0}\right)<\varepsilon$ for all $\lambda \in\left[\lambda_{0}-\delta, \lambda_{0}\right]$.

Now choose $n_{0}$ such that for every $n \geq n_{0}$, we have $\left|u_{n}^{-}\left(\lambda_{0}-\delta\right)-u^{-}\left(\lambda_{0}-\delta\right)\right|<\varepsilon$ and $\left|u_{n}^{-}\left(\lambda_{0}\right)-u^{-}\left(\lambda_{0}\right)\right|<\varepsilon$. Then, for all $n \geq n_{0}$ and all $\lambda \in\left[\lambda_{0}-\delta, \lambda_{0}\right]$, we obtain

$$
\begin{aligned}
0 & \leq u_{n}^{-}\left(\lambda_{0}\right)-u_{n}^{-}(\lambda) \leq u_{n}^{-}\left(\lambda_{0}\right)-u_{n}^{-}\left(\lambda_{0}-\delta\right) \\
& \leq\left|u_{n}^{-}\left(\lambda_{0}\right)-u^{-}\left(\lambda_{0}\right)\right|+\left|u^{-}\left(\lambda_{0}\right)-u^{-}\left(\lambda_{0}-\delta\right)\right|+\left|u^{-}\left(\lambda_{0}-\delta\right)-u_{n}^{-}\left(\lambda_{0}-\delta\right)\right| \leq 3 \varepsilon .
\end{aligned}
$$

The proof is completed by invoking the fact that $u_{n}^{-}$is left continuous at $\lambda_{0}$ for each $n<n_{0}$.

Theorem 3.6. For a subset $A$ of $\mathbb{E}^{1}$ the following conditions are equivalent:

(i) $\left.d_{\infty}\right|_{A}=\left.\tau_{\ell}\right|_{A}$;

(ii) for each sequence $\left\{u_{n}\right\}_{n=1}^{\infty}=\left\{\left(u_{n}^{-}, u_{n}^{+}\right)\right\}_{n \geq 1} \subset A$ which levelly converges to an element $\left(u^{-}, u^{+}\right)$in $A$, we have $\lim _{n} u_{n}^{-}\left(\lambda_{+}\right)=u^{-}\left(\lambda_{+}\right)$and $\lim _{n} u_{n}^{+}\left(\lambda_{+}\right)=u^{+}(\lambda+)$ for all $\lambda \in[0,1[$; 
(iii) every $\tau_{\ell}$-convergent sequence $\left\{u_{n}\right\}_{n=1}^{\infty} \subset A$ is right equicontinuous.

Proof. (i) $\Rightarrow$ (ii) easily follows from the definition of uniform convergence and (ii) $\Rightarrow$ (iii) runs along similar lines to the ones used in Lemma 3.5. To see (iii) $\Rightarrow(\mathrm{i})$, we need to prove that the identity map from $\left(\mathbb{E}^{1}, \tau_{\ell}\right)$ onto $\left(\mathbb{E}^{1}, \tau_{u}\right)$ is continuous, that is, if $\left\{\left(u_{n}^{-}, u_{n}^{+}\right)\right\}_{n>1}$ converges to $\left(u^{-}, u^{+}\right)$in $\left(\mathbb{E}^{1}, \tau_{\ell}\right)$, then $\left\{\left(u_{n}^{-}, u_{n}^{+}\right)\right\}_{n \geq 1} d_{\infty}$-converges to $\left(u^{-}, u^{+}\right)$. Assume that $\left\{\left(u_{n}^{-}, u_{n}^{+}\right)\right\}_{n \geq 1}$ does not $d_{\infty}$-converge to $\left(u^{-}, u^{+}\right)$. Then we can clearly assume that $\left\{u_{n}^{-}\right\}_{n \geq 1}$ does not converge uniformly to $u^{-}$. Under this assumption, we can find $\varepsilon>0$, an infinite sequence of natural numbers $n_{1}<n_{2}<n_{3}<\cdots$ and a sequence $\left\{\lambda_{n_{k}}\right\}_{k \in \mathbb{N}} \subset[0,1]$ such that $\left|u_{n_{k}}^{+}\left(\lambda_{n_{k}}\right)-u^{+}\left(\lambda_{n_{k}}\right)\right| \geq 3 \varepsilon$. Let us suppose, with no loss of generality that the sequence $\left\{\lambda_{n_{k}}\right\}_{k \in \mathbb{N}}$ converges to $\left.\left.\lambda_{0} \in\right] 0,1\right]$. Notice that $\left\{\lambda_{n_{k}}\right\}_{k \in \mathbb{N}}$ has infinite many different elements because $\left\{u^{-}\right\}_{\geq 1}$ pointwise converges to $u^{-}$. Assume that there exists an infinite subsequence of $\left\{\lambda_{n_{k}}\right\}_{k \in \mathbb{N}}$ whose elements are greater than $\lambda_{0}$. For simplicity, we shall denote this subsequence again by $\left\{\lambda_{n_{k}}\right\}_{k \in \mathbb{N}}$. Our hypothesis and the fact that $u^{-}$and the elements of $\left\{u_{n}^{-}\right\}_{n=1}^{\infty}$ are right continuous at $\lambda_{0}$ allow us to find a sequence $\left\{n_{k}\right\}_{k}$ such that

$$
\begin{aligned}
\left|u_{n_{k}}^{+}\left(\lambda_{n_{k}}\right)-u^{+}\left(\lambda_{n_{k}}\right)\right| \leq & \left|u_{n_{k}}^{+}\left(\lambda_{n_{k}}\right)-u_{n_{k}}^{+}\left(\lambda_{0+}\right)\right| \\
& +\left|u_{n_{k}}^{+}\left(\lambda_{0+}\right)-u^{+}\left(\lambda_{0}+\right)\right|+\left|u^{+}\left(\lambda_{0}+\right)-u^{+}\left(\lambda_{n_{k}}\right)\right| \leq 3 \varepsilon,
\end{aligned}
$$

which leads us to a contradiction. On the other hand, if there is an infinite subsequence of $\left\{\lambda_{n_{k}}\right\}_{k \in \mathbb{N}}$ whose elements are less than $\lambda_{0}$, by means of Lemma 3.5, an argument similar to the previous one also permits us to obtain a contradiction. Thus, the proof is complete.

We finish this section by giving some topological properties of $\left(\mathbb{E}^{1}, \tau_{\ell}\right)$ and the underlying topological space $\left(\widehat{\mathbb{E}^{1}}, \tau_{p}\right)$ of the uniform space $\left(\widehat{\mathbb{E}^{1}}, \widehat{\mathcal{u}}\right)$. Recall that a topological space is said to be hemicompact if it has a sequence of compact subsets such that every compact subset of the space lies inside some compact set in the sequence. The space is said to be Lindelöf, if every open cover has a countable subcover.

Theorem 3.7. $\left(\widehat{\mathbb{E}^{1}}, \tau_{p}\right)$ is a hemicompact space.

Proof. Let $K_{n}$ denote the set of all $u \in \widehat{\mathbb{E}^{1}}$ such that $\left|u^{-}(0)-u^{+}(0)\right| \leq n$. It is clear that these sets are pointwise closed and pointwise bounded so that they are compact subsets of $(\mathbb{R} \times \mathbb{R})^{[0,1]}$. Since $\widehat{\mathbb{E}^{1}}$ is a closed subset of $(\mathbb{R} \times \mathbb{R})^{[0,1]}$, they are compact subsets of $\left(\widehat{\mathbb{E}^{1}}, \tau_{p}\right)$. Now, given a compact subset $K$ of $\left(\widehat{\mathbb{E}^{1}}, \tau_{p}\right)$, there exists a constant $C>0$ such that $\left|u^{-}(0)\right|,\left|u^{+}(0)\right| \leq C$. Thus, $\left|u^{-}(0)-u^{+}(0)\right| \leq 2 C$. Thus, if $n>2 C, K \subseteq K_{n}$. This completes the proof.

Hemicompact spaces are Lindelöf, and every Lindelöf space is strongly paracompact ([10, Theorem 5.3.11]) so that we conclude the following.

Corollary 3.8. $\left(\widehat{\mathbb{E}^{1}}, \tau_{p}\right)$ is a Lindelöf space. In particular, it is strongly paracompact and, consequently, a normal space.

Recall that a (strongly) paracompact space $X$ is Dieudonné complete, that is, the finest uniformity on $X$ is complete. Thus, the natural uniformity on $\mathbb{E}^{1}$ is not complete but the finest uniformity is. 
We close the section by showing that both $\left(\mathbb{E}^{1}, \tau_{\ell}\right)$ and $\left(\widehat{\mathbb{E}^{1}}, \tau_{p}\right)$ are Baire spaces, that is, the Baire category theorem holds in $\left(\mathbb{E}^{1}, \tau_{\ell}\right)$ and in $\left(\widehat{\mathbb{E}^{1}}, \tau_{p}\right)$ : for each sequence $G_{1}, G_{2}, \ldots$ of open dense subsets of $\left(\mathbb{E}^{1}, \tau_{\ell}\right)$ (resp., of $\left(\widehat{\mathbb{E}^{1}}, \tau_{p}\right)$ ) the intersection $\bigcap_{n \geq 1} G_{n}$ is a dense set.

Theorem 3.9. $\left(\mathbb{E}^{1}, \tau_{\ell}\right)$ and $\left(\widehat{\mathbb{E}^{1}}, \tau_{p}\right)$ are Baire spaces.

Proof. Since a topological space $X$ that contains a dense subspace which is a Baire space is a Baire space itself $([10,3.9 . J(b)])$, it suffices to prove that $\left(\mathbb{C}^{1}, \tau_{\ell}\right)$ is a Baire space. To see this, first recall that the topologies $\tau_{d_{\infty}}$ and $\tau_{\ell}$ coincide in $\mathbb{C}^{1}$. Next, notice that the space $\left(\mathbb{C}^{1}, d_{\infty}\right)$ is a metric complete space and apply that metric complete spaces are Baire spaces.

\section{An Arzela-Ascoli Theorem for $C\left(X,\left(\mathbb{E}^{1}, \tau_{l}\right)\right)$}

In this section, $X$ will denote a locally compact topological space. We shall assume that the space $C\left(X,\left(\mathbb{E}^{1}, \tau_{l}\right)\right)$ of $\mathbb{E}^{1}$-valued continuous functions is endowed with the compact-open topology and given a subset $F$ of $C\left(X,\left(\mathbb{E}^{1}, \tau_{l}\right)\right)$, we shall write $F[x]:=\{f(x): f \in F\}$ for any $x \in X$.

As in [12], we have the following.

Theorem 4.1. Let $F \subset C\left(X,\left(\mathbb{E}^{1}, \tau_{l}\right)\right)$. Then $F$ is compact if and only if

(i) $F$ is closed;

(ii) For any $x \in X, F[x]$ is pointwise closed and uniformly support-bounded;

(iii) $F$ is evenly equicontinuous. as follows.

When we deal with $C\left(X,\left(\mathbb{E}^{1}, \tau_{l}\right)\right)$, the definition of evenly continuous can be translated

Definition 4.2. A subset $F$ of $C\left(X,\left(\mathbb{E}^{1}, \tau_{l}\right)\right)$ is said to be evenly equicontinuous if for any $x_{0} \in$ $X, u \in \mathbb{E}^{1},\left\{\alpha_{1}, \ldots, \alpha_{n}\right\} \subset[0,1]$ and $\epsilon>0$, there exists an open neighborhood $V$ of $x_{0}$ and $\left\{\beta_{1}, \ldots, \beta_{m}\right\} \subset[0,1]$ such that if $f \in F$ and

$$
\max _{1 \leq i \leq m}\left\{\left|f\left(x_{0}\right)^{+}\left(\beta_{i}\right)-u^{+}\left(\beta_{i}\right)\right|,\left|f\left(x_{0}\right)^{-}\left(\beta_{i}\right)-u^{-}\left(\beta_{i}\right)\right|\right\}<\epsilon,
$$

then

$$
\max _{1 \leq i \leq n}\left\{\left|f(x)^{+}\left(\alpha_{i}\right)-u^{+}\left(\alpha_{i}\right)\right|,\left|f(x)^{-}\left(\alpha_{i}\right)-u^{-}\left(\alpha_{i}\right)\right|\right\}<\epsilon,
$$

for all $x \in V$.

For the sake of completeness, we give the proof of Theorem 4.1 following the same pattern as in [12].

Proof of Theorem 4.1. We have the following. 
Necessity. Let us suppose that $F$ is compact in the compact-open topology of $C\left(X,\left(\mathbb{E}^{1}, \tau_{l}\right)\right)$.

(i) $F$ is closed since $C\left(X,\left(\mathbb{E}^{1}, \tau_{l}\right)\right)$ is Hausdorff as $\left(\mathbb{E}^{1}, \tau_{l}\right)$ is so $([7])$.

(ii) It is apparent that, for any $x \in X$, the map $L_{x}: C\left(X,\left(\mathbb{E}^{1}, \tau_{l}\right)\right) \rightarrow\left(\mathbb{E}^{1}, \tau_{l}\right)$ defined to be $L_{x}(f):=f(x)$ is continuous. Hence, since $F$ is compact, then $L_{x}(F)=F[x]$ is compact in $\left(\mathbb{E}^{1}, \tau_{l}\right)$ for each $x \in K$. Therefore, by Theorem $2.3, F[x]$ is, for any $x \in K$, pointwise closed and uniformly support bounded.

(iii) Fix $x_{0} \in K, u \in \mathbb{E}^{1},\left\{\alpha_{1}, \ldots, \alpha_{n}\right\} \subset[0,1]$ and $\epsilon>0$. Let $\left\{\beta_{1}, \ldots, \beta_{m}\right\} \subset[0,1]$ such that $\left\{\alpha_{1}, \ldots, \alpha_{n}\right\} \subseteq\left\{\beta_{1}, \ldots, \beta_{m}\right\}$. Let $F^{\prime} \subseteq F$ such that

$$
\max _{1 \leq i \leq m}\left\{\left|f\left(x_{0}\right)^{+}\left(\beta_{i}\right)-u^{+}\left(\beta_{i}\right)\right|,\left|f\left(x_{0}\right)^{-}\left(\beta_{i}\right)-u^{-}\left(\beta_{i}\right)\right|\right\} \leq \epsilon,
$$

for all $f \in F^{\prime}$. It is apparent that $F^{\prime}$ is closed and, consequently, it is compact. Let us consider the continuous map $L(f, x):=f(x)$ defined from $F \times X$ to $\mathbb{E}^{1}$. Then

$$
L\left(F^{\prime} \times\left\{x_{0}\right\}\right) \subset U:=\left\{v \in \mathbb{E}^{1}: \max _{1 \leq i \leq n}\left\{\left|v^{+}\left(\alpha_{i}\right)-u^{+}\left(\alpha_{i}\right)\right|,\left|v^{-}\left(\alpha_{i}\right)-u^{-}\left(\alpha_{i}\right)\right|\right\}<\epsilon\right\},
$$

which is to say that the compact subset $F^{\prime} \times\left\{x_{0}\right\}$ is included in $L^{-1}(V)$. By Wallace theorem ([10, Theorem 3.2.10]), there exists an open neighborhood, $V$, of $x_{0}$ such that $f(x) \in U$ for all $f \in F^{\prime}$ and all $x \in V$, that is,

$$
\max _{1 \leq i \leq n}\left\{\left|f(x)^{+}\left(\alpha_{i}\right)-u^{+}\left(\alpha_{i}\right)\right|,\left|f(x)^{-}\left(\alpha_{i}\right)-u^{-}\left(\alpha_{i}\right)\right|\right\}<\epsilon .
$$

Sufficiency. Let us first check that $\bar{F}$, the closure of $F$ in the pointwise convergence topology, is also evenly equicontinuous. Fix $x_{0} \in K, u \in \mathbb{E}^{1},\left\{\alpha_{1}, \ldots, \alpha_{n}\right\} \subset[0,1]$ and $\epsilon>0$. Then, by the evenly equicontinuity of $F$, there exists a neighborhood $V$ of $x_{0}$ and $\left\{\beta_{1}, \ldots, \beta_{m}\right\} \subset[0,1]$ such that if $f \in F$ and

$$
\max _{1 \leq i \leq m}\left\{\left|f\left(x_{0}\right)^{+}\left(\beta_{i}\right)-u^{+}\left(\beta_{i}\right)\right|,\left|f\left(x_{0}\right)^{-}\left(\beta_{i}\right)-u^{-}\left(\beta_{i}\right)\right|\right\}<\epsilon,
$$

then

$$
\max _{1 \leq i \leq n}\left\{\left|f(x)^{+}\left(\alpha_{i}\right)-u^{+}\left(\alpha_{i}\right)\right|,\left|f(x)^{-}\left(\alpha_{i}\right)-u^{-}\left(\alpha_{i}\right)\right|\right\}<\epsilon,
$$

for all $x \in V$. Let us take $g \in \bar{F}$ such that

$$
\max _{1 \leq i \leq m}\left\{\left|g\left(x_{0}\right)^{+}\left(\beta_{i}\right)-u^{+}\left(\beta_{i}\right)\right|,\left|g\left(x_{0}\right)^{-}\left(\beta_{i}\right)-u^{-}\left(\beta_{i}\right)\right|\right\}<\epsilon .
$$

We must check

$$
\max _{1 \leq i \leq n}\left\{\left|g(x)^{+}\left(\alpha_{i}\right)-u^{+}\left(\alpha_{i}\right)\right|,\left|g(x)^{-}\left(\alpha_{i}\right)-u^{-}\left(\alpha_{i}\right)\right|\right\}<\epsilon
$$


for all $x \in V$. To this end, let us choose a net $\left\{g_{n}\right\}$ in $F$ which converges pointwise to $g$. Hence, eventually,

$$
\max _{1 \leq i \leq m}\left\{\left|g_{n}\left(x_{0}\right)^{+}\left(\beta_{i}\right)-u^{+}\left(\beta_{i}\right)\right|,\left|g_{n}\left(x_{0}\right)^{-}\left(\beta_{i}\right)-u^{-}\left(\beta_{i}\right)\right|\right\}<\epsilon .
$$

Consequently, by the evenly equicontinuity of $F$,

$$
\max _{1 \leq i \leq n}\left\{\left|g_{n}(x)^{+}\left(\alpha_{i}\right)-u^{+}\left(\alpha_{i}\right)\right|,\left|g_{n}(x)^{-}\left(\alpha_{i}\right)-u^{-}\left(\alpha_{i}\right)\right|\right\}<\epsilon
$$

for all $x \in V$, which yields the desired fact.

As in the necessity case, let us consider the map $L(f, x):=f(x)$ defined from $\bar{F} \times X$ to $\mathbb{E}^{1}$. We claim that $L$ is continuous. To this end, fix $x_{0} \in K, u \in \mathbb{E}^{1},\left\{\alpha_{1}, \ldots, \alpha_{n}\right\} \subset[0,1]$ and $\epsilon>0$. Since $\bar{F}$ is evenly equicontinuous, there exists an open neighborhood $V$ of $x_{0}$ and $\left\{\beta_{1}, \ldots, \beta_{m}\right\} \subset[0,1]$ such that if $f \in W:=\left\{f \in \bar{F}: \max _{1 \leq i \leq m}\left\{\left|f\left(x_{0}\right)^{+}\left(\beta_{i}\right)-u^{+}\left(\beta_{i}\right)\right|, \mid f\left(x_{0}\right)^{-}\left(\beta_{i}\right)-\right.\right.$ $\left.\left.u^{-}\left(\beta_{i}\right) \mid\right\}<\epsilon\right\}$, then $L(W \times V) \subset U$, where

$$
U:=\left\{v \in \mathbb{E}^{1}: \max _{1 \leq i \leq n}\left\{\left|v^{+}\left(\alpha_{i}\right)-u^{+}\left(\alpha_{i}\right)\right|,\left|v^{-}\left(\alpha_{i}\right)-u^{-}\left(\alpha_{i}\right)\right|\right\}<\epsilon\right\} .
$$

Since it is also clear that $W$ is open in $\bar{F}$ and $U$ in open in $\left(\mathbb{E}^{1}, \tau_{l}\right)$, then $L$ is continuous.

Similarly, by the evenly equicontinuity of $\bar{F}$, it can be deduced that if $f \in \bar{F}$, then $f \in C\left(X,\left(\mathbb{E}^{1}, \tau_{l}\right)\right)$.

Next, we shall check that the closure of $F$ in the pointwise convergence topology, $\bar{F}$, coincides with the closure of $F$ in the compact-open topology, $\mathrm{cl}(F)$. It suffices to check that, in this context, the topology of pointwise convergence is larger than the compact-open topology. Thus, it is well known that, given a compact subset $K_{1}$ of $K, u_{0} \in \mathbb{E}^{1}$, and $\left\{\alpha_{1}, \ldots, \alpha_{n}\right\} \subset[0,1]$, the set $B\left(K_{1},\left\{\alpha_{1}, \ldots, \alpha_{n}\right\}, u_{0}\right)$ given by

$$
\left\{g \in \bar{F}: \max _{1 \leq i \leq n}\left\{\left|g(x)^{+}\left(\alpha_{i}\right)-u_{0}^{+}\left(\alpha_{i}\right)\right|,\left|g(x)^{-}\left(\alpha_{i}\right)-u_{0}^{-}\left(\alpha_{i}\right)\right|\right\}<\epsilon \forall x \in K_{1}\right\}
$$

is a member of a subbase for the compact-open topology in $C\left(X,\left(\mathbb{E}^{1}, \tau_{l}\right)\right)$.

Fix $f \in B\left(K_{1},\left\{\alpha_{1}, \ldots, \alpha_{n}\right\}, u_{0}\right)$. Then $\{f\} \times K_{1}$, which is compact in $\bar{F} \times K$, belongs to $L^{-1}(U)$, where

$$
U:=\left\{v \in \mathbb{E}^{1}: \max _{1 \leq i \leq n}\left\{\left|v^{+}\left(\alpha_{i}\right)-u_{0}^{+}\left(\alpha_{i}\right)\right|,\left|v^{-}\left(\alpha_{i}\right)-u_{0}^{-}\left(\alpha_{i}\right)\right|\right\}<\epsilon\right\} .
$$

From the previous paragraphs, $L^{-1}(U)$ is open. Hence, by Wallace theorem ([10, Theorem 3.2.10]), there exists an open neighborhood, $N$, of $f$ in the pointwise convergence topology such that $N \times K_{1} \in L^{-1}(U)$, which is to say that $N \subset B\left(K_{1},\left\{\alpha_{1}, \ldots, \alpha_{n}\right\}, u_{0}\right)$. This means that the topology of pointwise convergence is larger than the compact-open topology.

Finally, we claim that $\mathrm{cl}(F)$ is compact in the compact-open topology. Indeed, we shall check that it is compact in the topology of pointwise convergence, but we have already 
checked that both topologies coincide on $\bar{F}=\mathrm{cl}(F)$. To this end, we can consider $\bar{F}$ as a closed subset of the product $\prod_{x \in K}\{\overline{F[x]}\}$. By Tychonoff's theorem, $\prod_{x \in K}\{\overline{F[x]}\}$ is compact in the topology of pointwise convergence since, by Theorem 2.3, $F[x]$ is compact for every $x \in K$. Thus, $\bar{F}$ is compact and, since by hypothesis, $F$ is closed, then $F$ is compact, as was proved.

\section{Acknowledgments}

This research is supported by the Spanish Ministry of Science and Education (Grant nos. MTM2011-22457 and MTM2011-23118) and by Bancaixa (Project P11B2011-30).

\section{References}

[1] S. S. L. Chang and L. A. Zadeh, “On fuzzy mapping and control," IEEE Transactions on Systems, Man, and Cybernetics, vol. 2, pp. 30-34, 1972.

[2] P. Diamond and P. Kloeden, Metric Spaces of Fuzzy Sets-Theory and Applications, World Scientific, Singapore, 1994.

[3] P. Diamond and P. Kloeden, "Metric topology of fuzzy numbers and fuzzy analysis," in Fundamentals of Fuzzy Sets, vol. 1 of Handbook Series of Fuzzy Sets, pp. 583-641, Kluwer, Dordrecht, The Netherlands, 2000.

[4] R. Goetschel, and W. Voxman, "Elementary fuzzy calculus," Fuzzy Sets and Systems, vol. 18, no. 1, pp. 31-43, 1986.

[5] P. Diamond and P. Kloeden, "Characterization of compact subsets of fuzzy sets," Fuzzy Sets and Systems, vol. 29, no. 3, pp. 341-348, 1989.

[6] C. X. Wu and G. X. Wang, "Convergence of sequences of fuzzy numbers and fixed point theorems for increasing fuzzy mappings and application," Fuzzy Sets and Systems, vol. 130, no. 3, pp. 383-390, 2002.

[7] J.-X. Fang and H. Huang, "Some properties of the level convergence topology on fuzzy number space $E^{n}, "$ Fuzzy Sets and Systems, vol. 140, no. 3, pp. 509-517, 2003.

[8] D. S. Okhezin, "Study of families of monotone continuous functions on Tychonoff spaces," Journal of Mathematical Sciences, vol. 144, no. 34, pp. 4152-4183, 2007.

[9] O. Kaleva and S. Seikkala, “On fuzzy metric spaces,” Fuzzy Sets and Systems, vol. 12, no. 3, pp. 215-229, 1984.

[10] R. Engelking, General topology, vol. 6 of Sigma Series in Pure Mathematics, Heldermann, Berlin, Germany, 2nd edition, 1989.

[11] J. J. Font and M. Sanchis, "Compact subsets in the fuzzy numberspace," submitted.

[12] J. L. Kelley, General Topology, vol. 27 of Graduate Texts in Mathematics, Springer, New York, NY, USA, 1975. 


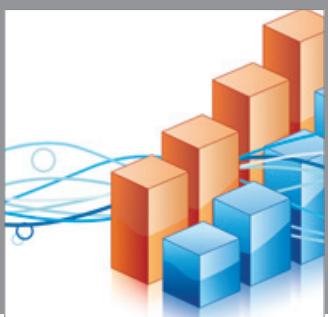

Advances in

Operations Research

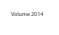

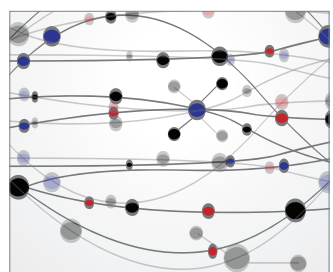

\section{The Scientific} World Journal
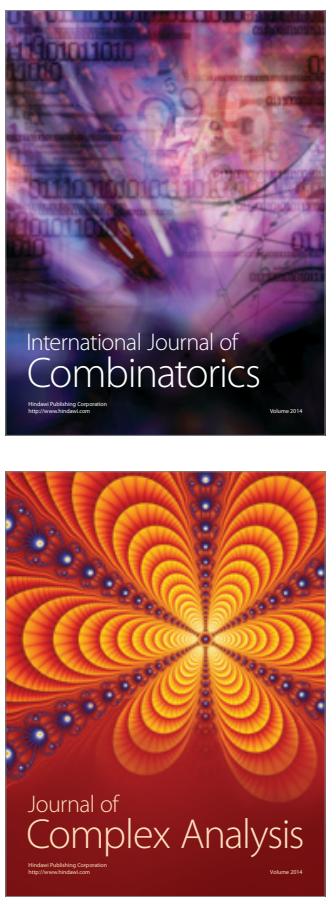

International Journal of

Mathematics and

Mathematical

Sciences
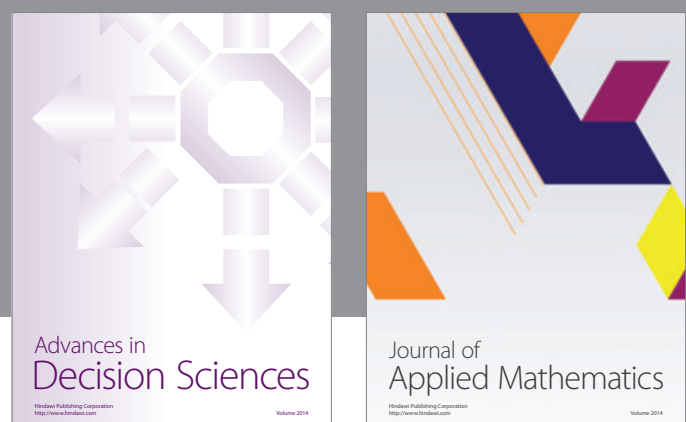

Journal of

Applied Mathematics
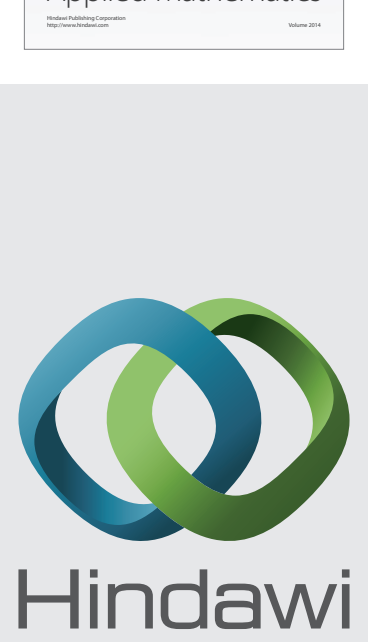

Submit your manuscripts at http://www.hindawi.com
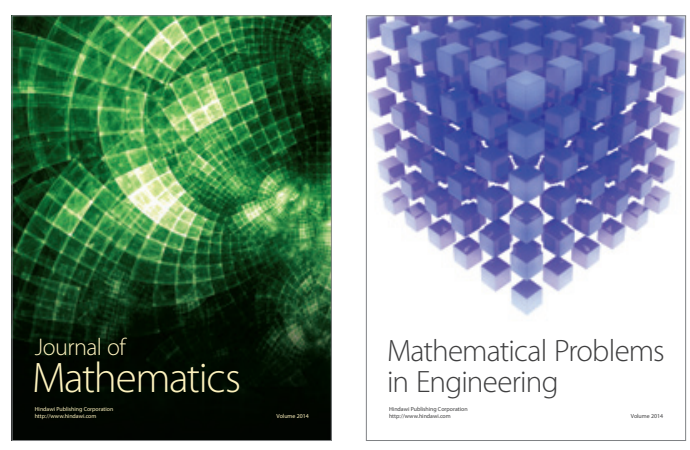

Mathematical Problems in Engineering
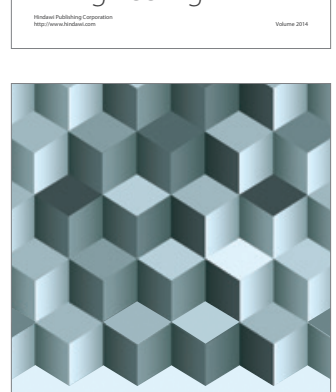

Journal of

Function Spaces
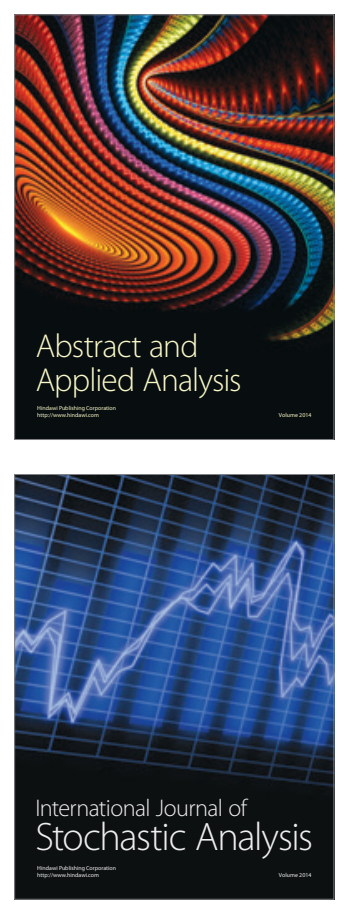

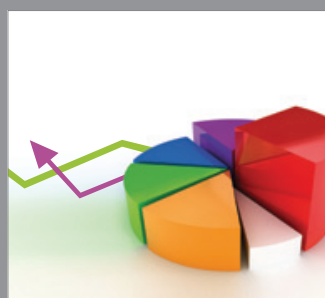

ournal of

Probability and Statistics

Promensencen
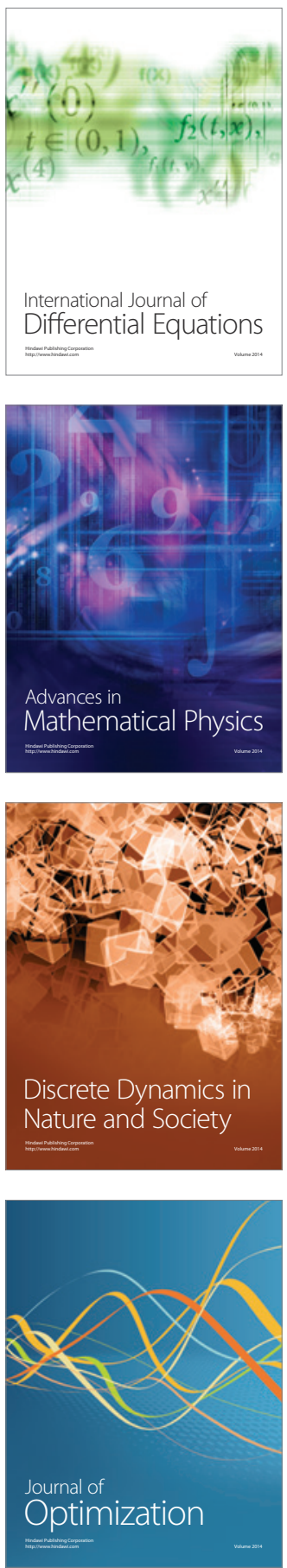